Correction

\title{
Correction: Rasheduzzaman, M., et al. Reverse QMRA as a Decision Support Tool: Setting Acceptable Concentration Limits for Pseudomonas aeruginosa and Naegleria fowleri. Water 2019, 11, 1850
}

\author{
Md Rasheduzzaman ${ }^{1, * \mathbb{D}}$, Rajveer Singh ${ }^{1} \mathbb{C}$, Charles N. Haas ${ }^{1}$, Dienye Tolofari ${ }^{1}$, \\ Hamed Yassaghi ${ }^{1}{ }^{\mathbb{D}}$, Kerry A. Hamilton ${ }^{2}$, Zhao Yang ${ }^{1}$ and Patrick L. Gurian ${ }^{1}$ \\ 1 Department of Civil, Architectural and Environmental Engineering, Drexel University, 3141 Chestnut Street, \\ Philadelphia, PA 19104, USA; rs443@drexel.edu (R.S.); haas@drexel.edu (C.N.H.); dlt76@drexel.edu (D.T.); \\ hy387@drexel.edu (H.Y.); zy334@drexel.edu (Z.Y.); plg28@drexel.edu (P.L.G.) \\ 2 The Biodesign Institute Center for Environmental Health Engineering \& The School of Sustainable \\ Engineering and the Built Environment, Arizona State University, Tempe, AZ 85281, USA; \\ kerry.hamilton@asu.edu \\ * Correspondence: mr3349@drexel.edu; Tel.: +1-215-895-2889
}

Received: 6 November 2019; Accepted: 7 November 2019; Published: 28 November 2019

The authors regret to report that the paper "Reverse QMRA as a Decision Support Tool: Setting Acceptable Concentration Limits for Pseudomonas aeruginosa and Naegleria fowleri" contains some erroneous computations.

An error in the supplementary Table S2 occurred due to confusion between the 'natural log' versus 'base 10 ' conversion. The errors do not influence the larger message of the study but do result in lower target concentrations for $N$. fowleri.

The authors wish to make the following corrections to this paper [1]:

In the 'Abstract' Section:

1. Thirteenth sentence (lines 13-15)

For N. fowleri, based on the DALY approach, critical concentrations were 0.000030 N. fowleri/L for swimming and $0.00000060 \mathrm{~N}$. fowleri/L for neti pot ${ }^{\mathrm{TM}}$ use scenario.

2. Seventeenth sentence (lines 17-18)

For N. fowleri, the $10^{-4}$ annual risk target approach resulted in 0.022 N. fowleri/L and the DALY approach resulted in $0.00000064 \mathrm{~N}$. fowleri/L for the neti pot $^{\mathrm{TM}}$ scenario.

In the 'Materials and Methods' Section:

1. $\quad 2.1$ Risk Characterization, Equation (2) descriptions

where, $D W$ is the disability weight, $L_{L}$ is the average duration of the case until death (years) and $L_{D}$ is years lived with infection/illness for non-fatal cases.

2. Table 3 
Table 3. Monte Carlo input parameters for P. aeruginosa and N. fowleri.

\begin{tabular}{|c|c|c|c|c|}
\hline Parameter & Unit & Value & Distribution & Source \\
\hline \multicolumn{5}{|c|}{ P. aeruginosa } \\
\hline alpha & & $\begin{array}{c}2.5 \%=0.115 ; 5 \%=0.124 \\
95 \%=0.386\end{array}$ & Gamma & [49] \\
\hline $\mathrm{N}_{50}$ & & $\begin{array}{c}2.5 \%=4730 ; 5 \%=6010 \\
95 \%=70,500\end{array}$ & Gamma & [49] \\
\hline Contact (Drop*) & $\mathrm{mL} /$ use & $\begin{array}{c}\text { Likeliest }=0.05 ; \operatorname{Min}=0.01 ; \\
\operatorname{Max}=0.15\end{array}$ & Triangular & Assumption \\
\hline Contact (E.M. $\left.{ }^{\Phi}\right)$ & $\mathrm{mL} /$ use & $\begin{array}{c}\text { Likeliest }=0.062 ; \operatorname{Min}= \\
0.052 ; \operatorname{Max}=0.07\end{array}$ & Triangular & $\begin{array}{l}\text { Measured by this } \\
\text { study }\end{array}$ \\
\hline \multicolumn{5}{|c|}{ N. fowleri } \\
\hline alpha & & $\begin{array}{c}2.5 \%=0.84 ; 5 \%=0.93 ; 95 \% \\
=72\end{array}$ & Gamma & Fit by this study \\
\hline $\mathrm{N}_{50}$ & & $\begin{array}{c}2.5 \%=82 ; 5 \%=92 ; 95 \%= \\
254\end{array}$ & Gamma & \\
\hline $\begin{array}{c}\text { Contact } \\
\text { (Swimming) }\end{array}$ & $\mathrm{mL} /$ use & $\begin{array}{c}\text { Likeliest }=1 ; \operatorname{Min}=0.1 \\
\operatorname{Max}=10\end{array}$ & Triangular & Assumption \\
\hline $\begin{array}{c}\text { Contact } \\
\left(\text { Neti Pot }^{\mathrm{TM}}\right)\end{array}$ & $\mathrm{mL} / \mathrm{use}$ & $\begin{array}{c}\text { Likeliest }=180 ; \operatorname{Min}=120 ; \\
\operatorname{Max}=240\end{array}$ & Triangular & [48] \\
\hline
\end{tabular}

Notes: Drop* represents the contact rate based on the drop scenarios; E.M. ${ }^{\Phi}$ represents the contact rate based on the experimental measurements of water volume in contact lenses.

In the 'Results' Section:

1. $\quad$ 3.1 Dose-Response Model, first paragraph (lines 1-3)

The details of the goodness of fit (i.e., deviance and combined AIC values) for various dose-response models for N. fowleri are shown in Table 4.

2. Table 4

Third column heading "MLE Estimate" should be changed to "Deviance".

3. 3.1 Dose-Response Model, second paragraph (lines 2-5)

The maximum likelihood estimates for beta-Poisson model parameters alpha $(\alpha)$ and $\mathrm{N}_{50}$ were found to be 1.59 and 156, respectively, from 10,000 bootstrap iterations. The $95 \%$ confidence interval for alpha $(\alpha)$ was $(0.93,72)$ and the $95 \%$ confidence interval for $\mathrm{N}_{50}$ was $(92,254)$.

4. $\quad 3.2$ Risk Characterization, first paragraph (lines 4-6)

For $P$. aeruginosa, the risk of illness per event calculated based on DALY was $2.81 \times 10^{-9}$, which was two orders of magnitude lower than the per event risk of illness based on the annual risk metric $\left(2.74 \times 10^{-7}\right)$.

5. Table 5

6. $\quad 3.2$ Risk Characterization, second paragraph (lines 1-5)

For N. fowleri, the risk of death for neti pot ${ }^{\mathrm{TM}}$ uses per event calculated based on DALYs was 4.77 $\times 10^{-10}$, which was five orders of magnitude lower than the per event risk of death calculated based on annual risk metric $\left(1.67 \times 10^{-5}\right)$. Similarly, for $N$. fowleri exposure during swimming, DALY and annual risk based per event risks followed the same pattern as neti pot ${ }^{\mathrm{TM}}$ exposure, i.e., a five orders of magnitude difference in the risk of death.

7. 3.3 Concentrations, second paragraph (lines $2-4$ )

The highest critical concentration for $N$. fowleri was obtained for the swimming scenario using the annual risk metric (mean concertation $1.4 \mathrm{~N}$. fowleri/L with a $95 \%$ confidence interval of $(0.29,4.0)$ ).

8. 3.3 Concentrations, third paragraph (lines 1-3) 
Similarly, the concentration for the neti pot $^{\mathrm{TM}}$ scenario was found to be $0.021 \mathrm{~N}$. fowleri/L with a $95 \%$ confidence interval of $(0.010,0.034)$ for the annual risk approach. Based on the DALY approach, limits of concentrations were $0.000030 \mathrm{~N}$. fowleri/L for swimming and $0.00000060 \mathrm{~N}$. fowleri/L for neti pot $^{\mathrm{TM}}$ use scenario.

9. Table 6

10. 3.3 Concentrations, fourth paragraph (lines $2-4$ )

Considering the hot water scenario, the annual risk approach resulted in $0.022 \mathrm{~N}$. fowleri/L, and the DALY approach resulted in 0.00000064 N. fowleri/L for the neti pot ${ }^{\mathrm{TM}}$ scenario (Scenario 5).

Table 5. Risk of illness/death based on DALYs and annual risk approach.

\begin{tabular}{ccccc}
\hline \multirow{2}{*}{ Approach } & \multirow{2}{*}{ Risk of Illness/Death } & P. aeruginosa & \multicolumn{2}{c}{ N. fowleri } \\
\cline { 3 - 5 } & & & Neti Pot $^{\text {TM }}$ & Swimming \\
\hline \multirow{2}{*}{ DALYs } & Annual & $1.03 \times 10^{-6}$ & $2.86 \times 10^{-9}$ & $2.45 \times 10^{-9}$ \\
& Per Event & $2.81 \times 10^{-9}$ & $4.77 \times 10^{-10}$ & $3.50 \times 10^{-10}$ \\
\hline \multirow{2}{*}{ Annual Risk } & Annual & $1.00 \times 10^{-4}$ & $1.00 \times 10^{-4}$ & $1.00 \times 10^{-4}$ \\
& Per Event & $2.74 \times 10^{-7}$ & $1.67 \times 10^{-5}$ & $1.43 \times 10^{-5}$ \\
\hline
\end{tabular}

Table 6. Concentration of P. aeruginosa and N. fowleri before and after heat inactivation ${ }^{1}$.

\begin{tabular}{|c|c|c|c|c|c|c|c|}
\hline \multirow{3}{*}{$\begin{array}{l}\text { Risk } \\
\text { Metric }\end{array}$} & \multirow{2}{*}{\multicolumn{2}{|c|}{$\begin{array}{l}\text { P. aeruginosa Conc. (CFU/L) } \\
(95 \% \mathrm{CI})\end{array}$}} & \multirow{2}{*}{\multicolumn{2}{|c|}{$\begin{array}{l}\text { N. fowleri Conc. }(N . \\
\text { fowleri } / \mathrm{L})(95 \% \mathrm{CI})\end{array}$}} & \multicolumn{3}{|c|}{ Heat Inactivation Conc. } \\
\hline & & & & & \multicolumn{2}{|c|}{ P. aeruginosa (CFU/L) } & \multirow{2}{*}{$\begin{array}{c}\begin{array}{c}\text { N. fowleri } \\
(\text { N. fowleri } / \mathrm{L})\end{array} \\
\begin{array}{c}\text { Neti Pot }{ }^{\mathrm{TM}} \\
\text { (Scenario 5) }\end{array}\end{array}$} \\
\hline & $\begin{array}{l}\text { Contact lens } \\
\text { (Drop) } \\
\text { (Scenario 1a) }\end{array}$ & $\begin{array}{l}\text { Contact lens } \\
\text { (E.M.) } \\
\text { (Scenario 1b) }\end{array}$ & $\begin{array}{l}\text { Neti Pot }{ }^{\mathrm{TM}} \\
\text { (Scenario 2) }\end{array}$ & $\begin{array}{l}\text { Swimming } \\
\text { (Scenario 3) }\end{array}$ & $\begin{array}{c}\text { Drop } \\
\text { (Scenario } \\
4 a)\end{array}$ & $\begin{array}{c}\text { E.M. } \\
\text { (Scenario } \\
4 b)\end{array}$ & \\
\hline DALY & $0.33(0.02-1.8)$ & $\begin{array}{c}0.30 \\
(0.02-0.99)\end{array}$ & $\begin{array}{c}0.00000060 \\
(0.00000030- \\
0.0000010)\end{array}$ & $\begin{array}{c}0.000030 \\
(0.000010- \\
0.00010)\end{array}$ & 0.60 & 0.55 & 0.00000064 \\
\hline $\begin{array}{l}\text { Annual } \\
\text { Risk }\end{array}$ & $33(2.0-118)$ & $30(2.3-100)$ & $\begin{array}{c}0.021 \\
(0.010-0.034)\end{array}$ & $1.4(0.29-4.0)$ & 61 & 55 & 0.022 \\
\hline
\end{tabular}

Notes: ${ }^{1}$ EPA has generally applied the 1 in 10,000 risk target to the risk of infection to provide a margin of safety that accounts for secondary transmission. In this study, neither the dose-response model provided infection as an endpoint (endpoint of the N. fowleri model was death and endpoint of the P. aeruginosa model was illness). If one wished to provide the additional margin of safety associated with infection as an endpoint, one would need to estimate the probability of illness/infection based on sources such as Cheng et al. (1999) [54] and multiply the concentrations provided in this study by that probability.

In the 'Discussion' Section:

1. $\quad$ Fifth paragraph (lines 3-5)

In general, the tolerable critical concentrations of $N$. fowleri are much lower compared to those for $P$. aeruginosa except for the swimming scenario with the annual risk metric which is still more than an order of magnitude below the $P$. aeruginosa values.

2. Fifth paragraph (lines 9-14)

The tolerable mean critical concentration of $N$. fowleri for the swimming scenario with the annual risk metric has a mean concentration 1.4 CFU/L, with a range from 0.29 to $4.0 \mathrm{CFU} / \mathrm{L}$. Given that the end response is death with a $100 \%$ percent mortality rate, it may be wise to pick a lower range of critical concentration $(0.29 \mathrm{CFU} / \mathrm{L})$ for developing standards, and the same can be applied for developing standards for other scenarios related to $N$. fowleri.

3. Fifth paragraph (lines 15-17) 
Considering 50 cells of $N$. fowleri and $200 \mathrm{~L}$ as a volume of water sample to be filtered, the detection limit of $N$. fowleri would be $0.25 \mathrm{~N}$. fowleri/L which is also aligned with our calculated boundary concentration of $N$. fowleri using the annual risk metric.

4. Sixth paragraph (lines 1-2)

Higher tolerable concentration standard can be set for using hot plumbing water for contact lenses exposure scenarios for P. aeruginosa but not for exposure scenarios for $N$. fowleri.

In the 'Acknowledgments' Section:

1. Please add this line after the last line of the current acknowledgments

The authors are grateful to Dr. Jade Mitchell and Kara Dean at Michigan State University for pointing out the errors in our dose-response parameters that prompted us to revise and correct the manuscript with updated results.

In the 'Supplementary Materials' Section:

1. Table S2

Table S2. Best fit parameters for the beta-Poisson model for N. fowleri, from 10,000 bootstrap iterations.

\begin{tabular}{cccccccc}
\hline \multirow{2}{*}{ Parameter MLE } & \multicolumn{7}{c}{ Percentiles } \\
\cline { 3 - 8 } & $\mathbf{0 . 5 \%}$ & $\mathbf{2 . 5 \%}$ & $\mathbf{5 \%}$ & $\mathbf{9 5 \%}$ & $\mathbf{9 7 . 5 \%}$ & $\mathbf{9 9 . 5 \%}$ \\
\hline Alpha & 1.59 & 0.72 & 0.84 & 0.93 & 72 & 1175 & 12946 \\
$\mathrm{~N}_{50}$ & 156 & 63 & 82 & 92 & 254 & 276 & 322 \\
\hline
\end{tabular}

The authors would like to apologize for any inconvenience caused to the readers by these changes. The manuscript will be updated, and the original will remain online on the article webpage, with a reference to this Correction.

\section{References}

1. Rasheduzzaman, M.; Singh, R.; Haas, C.N.; Tolofari, D.; Yassaghi, H.; Hamilton, K.A.; Yang, Z.; Gurian, P.L. Reverse QMRA as a Decision Support Tool: Setting Acceptable Concentration Limits for Pseudomonas aeruginosa and Naegleria fowleri. Water 2019, 11, 1850. [CrossRef]

(C) 2019 by the authors. Licensee MDPI, Basel, Switzerland. This article is an open access article distributed under the terms and conditions of the Creative Commons Attribution (CC BY) license (http://creativecommons.org/licenses/by/4.0/). 